\title{
Therapeutic effect of resveratrol on mice with depression
}

\author{
ZHENG GU, LIUJIE CHU and YAQIONG HAN \\ Department of Pharmacy, The Second Affiliated Hospital of Xinxiang Medical University, \\ Xinxiang, Henan 453002, P.R. China
}

Received September 17, 2018; Accepted February 8, 2019

DOI: $10.3892 /$ etm.2019.7311

\begin{abstract}
The effect and mechanism of resveratrol on depression-like behavior in mice with depression were investigated. A mouse model of depression was established by social isolation combined with chronic unpredictable stress. The mice were randomly divided into the control group, the model group, the low dose group, the medium dose group and the high dose group. The rats in the low, medium and high dose groups were intraperitoneally injected with resveratrol 10,20 and $30 \mathrm{mg} / \mathrm{kg}$, respectively. The control and model groups were intraperitoneally injected with an equal volume of normal saline. After 21 days of continuous treatment, the neurobehavioral changes of each group were analyzed by forced swimming test, tail suspension experiment and sucrose consumption experiment. Dopamine (DA) and serotoni (5-HT) and the level of brain-derived nerve growth factor (BDNF) in the prefrontal cortex of each group were analyzed by ELISA. The level of neuropeptide (NPY) expression was analyzed by western blot analysis. Compared with the model group, the immobility time of the tail suspension experiment and forced swimming experiment in the low, medium and high dose groups was significantly prolonged $(\mathrm{P}<0.05)$, while the $24 \mathrm{~h}$ sucrose consumption was significantly increased $(\mathrm{P}<0.05)$, showing a dose-dependent manner. Compared with the model group, the levels of DA and 5-HT in the prefrontal cortex of the low, medium and high dose groups were significantly increased, and showed a dose-dependent effect $(\mathrm{P}<0.05)$. Compared with the model group, the expression levels of NPY protein in the low, medium and high dose groups were significantly increased, and gradually increased with the increase of the dose, the difference was statistically significant $(\mathrm{P}<0.05)$. Resveratrol can significantly increase the levels of neurotransmitters DA and 5-HT in the prefrontal cortex and increase the expression of NPY in the brain, which can play an antagonistic role in depression.
\end{abstract}

Correspondence to: Dr Zheng Gu, Department of Pharmacy, The Second Affiliated Hospital of Xinxiang Medical University, 388 Jianshe Road, Muye, Xinxiang, Henan 453002, P.R. China E-mail: gwe65w@163.com

Key words: resveratrol, depression, neuropeptide Y, dopamine, serotonin

\section{Introduction}

Depression is a common chronic neurological disease. It has the characteristics of high prevalence, easy recurrence, disability, low rate of visiting and low treatment rate. It has become the fifth most serious disease affecting human health $(1,2)$. The occurrence of depression is the result of a combination of multiple factors, including heredity, personality, endocrine, living environment and disease. It is currently widely believed that the reduction in levels of neurotransmitters (dopamine and serotonin) and neurotrophic factors (neuropeptide Y) is closely related to the occurrence of depression (3-5). Therefore, it has been found that the purpose of alleviating or treating depression can be achieved by increasing the levels of neurotransmitters and neurotrophic factors. Resveratrol is a polyphenolic compound that is ubiquitous in plants. It has been found in modern pharmacological studies to have multiple biological functions such as antiinflammatory, anti-oxidation, antitumor, anti-thrombosis and anti-aging (6-10). Our early study found that resveratrol has antidepressant effects. This study analyzed its anti-depressant effects and possible mechanisms to provide a new approach to the treatment of depression.

\section{Materials and methods}

Experimental materials. A total of $50 \mathrm{SPF}$ grade C57BL/6 mice (female, 6-8 weeks old $20 \mathrm{~g}$ ) were purchased from Shanghai Slac Laboratory Animal Co., Ltd. (Shanghai, China); resveratrol was purchased from Sigma (USA); mouse dopamine (DA), serotonin (5-HT), brain-derived nerve growth factor (BDNF) and ELISA test kit were purchased from BioAssay Systems LLC (Hayward, CA, USA); Neuropeptide (NPY) and actin monoclonal antibodies were purchased from Santa Cruz, Biotechnology, Inc. (cat. nos. sc-133080 and sc-8432; Dallas, TX, USA); actin monoclonal antibody was purchased from Hangzhou Huaan Biological Co., Ltd. (Hangzhou, China); HRP-labled goat anti-mouse secondary antibody was purchased from Beijing Zhongshan Jinqiao Biological Co., Ltd. (cat. no. ZDR-5307; Beijing, China); RIPA tissue lysate was purchased from Beyotime Biotechnology Co., Ltd. (Nantong, China); chemiluminescence kit was purchased from Hangzhou Fude Biological Technology Co., Ltd. (Hangzhou, China); M200 full-band microplate reader was purchased from Tecan Group, Ltd. (Mannedorf, Switzerland). 
Construction and treatment of depression models. A model of depression was established using social isolation combined with chronic unpredictable stress. All modeled mice were housed individually and randomized alternating stress treatment was performed once a day for 21 days. The stressors were as follows: i) fasting for $24 \mathrm{~h}$; ii) water deprivation for $24 \mathrm{~h}$; iii) tilting squirrel cage for $30^{\circ}$ for $24 \mathrm{~h}$; iv) clipping $1 \mathrm{~cm}$ of the root of mouse tail for $1 \mathrm{~min}$; v) restraint with $9.5 \times 3 \mathrm{~cm}$ fixator for $2 \mathrm{~h}$; vi) continuously soaking in $17^{\circ} \mathrm{C}$ cold water for $3 \mathrm{~min}$; vii) continuously soaking in $40^{\circ} \mathrm{C}$ hot water for 3 min and viii) day alternates with night. Each stimulus was not used continuously. After 21 days of stimulation, the mice were randomly divided into the model group, the low-dose group, the medium-dose group, and the high-dose group, with 10 mice in each group, and 10 mice of the same age were selected as controls. On the 11th day after stimulation, the low, medium and high dose groups were intraperitoneally injected with resveratrol 10, 20 and $30 \mathrm{mg} / \mathrm{kg}$, respectively. Both the control and model groups were intraperitoneally injected with an equal volume of physiological saline once a day. After 21 days of continuous treatment, the neurobehavioral changes of each group were analyzed by forced swimming test, tail suspension experiment and sucrose consumption test.

The study was approved by the Ethics Committee of The Second Affiliated Hospital of Xinxiang Medical University (Xinxiang, China).

Suspension experiment. The experiment was carried out in a plexiglass black box. The distal end of the mouse tail was fixed on a crossbar $30 \mathrm{~cm}$ from the ground. The head was facing down, and inverted status was maintained. After $6 \mathrm{~min}$, it was adapted for $2 \mathrm{~min}$, and then the immobility time was recorded. This result mainly reflected the degree of acquired helplessness in mice.

Forced swimming experiment. Two hours after receiving the drug treatment, the mice were placed in a glass cylinder of $30 \mathrm{~cm}$ in diameter and $25 \mathrm{~cm}$ in height, with a water depth of $15 \mathrm{~cm}$; The water temperature was maintained at $24^{\circ} \mathrm{C}$, and the mice were forced to soak for 6 min. Judging criteria: All mice showed only the head exposed horizontally, floating, and the limbs were still. The immobility time was recorded $4 \mathrm{~min}$ after soaking.

Sucrose consumption test. Each group of mice was fasted and water-deprived for $24 \mathrm{~h}$, then $100 \mathrm{ml} 30 \mathrm{mmol} / \mathrm{l}$ sucrose solution and $100 \mathrm{ml}$ purified water were returned to the squirrel cage at 10:00 a.m. the next day. After the mice were allowed to drink for $1 \mathrm{~h}$, the consumption of sucrose solution and water was measured. The sucrose partiality was then calculated according to the following formula. Sucrose partiality $(\%)=$ sucrose consumption/total liquid volume $\mathrm{x} 100 \%$.

Analysis of DA, 5-HT and BDNF levels by ELISA. After $24 \mathrm{~h}$ of the last administration, the mice were sacrificed. Brain tissue was taken, and $100 \mathrm{mg}$ was weighed. Physiological saline $(200 \mu \mathrm{l})$ was added, and the brain tissue was homogenized by a homogenizer, then centrifuged at $12,000 \mathrm{x}$ g and $4^{\circ} \mathrm{C}$ for $10 \mathrm{~min}$. The supernatant was taken, the levels of DA,
Table I. Comparison of behavioral indicators after treatment in each group of mice.

\begin{tabular}{lccc}
\hline Groups & $\begin{array}{c}\text { Tail } \\
\text { suspension } \\
\text { experiment(s) }\end{array}$ & $\begin{array}{c}\text { Forced } \\
\text { swimming } \\
\text { experiment(s) }\end{array}$ & $\begin{array}{c}\text { Sucrose } \\
\text { consumption } \\
\text { experiment(s) }\end{array}$ \\
\hline Control & $89.32 \pm 12.21$ & $79.33 \pm 14.20$ & $21.43 \pm 6.17$ \\
Model & $271.43 \pm 20.19^{\mathrm{a}}$ & $194.74 \pm 21.09^{\mathrm{a}}$ & $8.43 \pm 3.18^{\mathrm{a}}$ \\
Low-dose & $221.33 \pm 17.17^{\mathrm{b}}$ & $170.32 \pm 18.39^{\mathrm{b}}$ & $17.11 \pm 4.21^{\mathrm{b}}$ \\
Medium-dose & $174.21 \pm 14.12^{\mathrm{b}, \mathrm{c}}$ & $141.30 \pm 16.33^{\mathrm{b}, \mathrm{c}}$ & $14.20 \pm 3.99^{\mathrm{b}, \mathrm{c}}$ \\
High-dose & $130.39 \pm 15.18^{\mathrm{b}-\mathrm{d}}$ & $129.43 \pm 16.01^{\mathrm{b}-\mathrm{d}}$ & $11.98 \pm 3.02^{\mathrm{b}-\mathrm{d}}$
\end{tabular}

${ }^{\mathrm{a}} \mathrm{P}<0.05$ compared with the control group; ${ }^{\mathrm{b}} \mathrm{P}<0.05$ compared with the model group; ${ }^{\mathrm{C}}<0.05$ compared with the low-dose group; ${ }^{\mathrm{d}} \mathrm{P}<0.05$ compared with the medium-dose group.

5-HT and BDNF were detected according to the manufacturer's instructions of ELISA.

Western blot analysis of NPY protein expression levels. The mice were sacrificed $24 \mathrm{~h}$ after the last administration; brain tissue was taken, and $100 \mathrm{mg}$ was weighed. Tissue lysate $(200 \mu \mathrm{l})$ was added, and homogenized by homogenizer; allowed to stand on ice for $30 \mathrm{~min}$, and centrifuged at $12,000 \mathrm{x} \mathrm{g}$ for $30 \mathrm{~min}$ at $4^{\circ} \mathrm{C}$. Loading buffer $5 \mathrm{X}(50 \mu 1$, $60 \mathrm{mM}$ Tris-HCl pH6.8, $2 \%$ SDS, $0.1 \%$ bromophenol blue, $25 \%$ glycerol; $14 \mathrm{mM} \beta$-mercaptoethanol) was added to the supernatant, boiled for $20 \mathrm{~min}$ in a metal bath, then SDS-PAGE was performed. After electrophoresis, the gel transferred to PVDF membrane, blocked with 5\% skim milk powder for $1 \mathrm{~h}$, and then incubated overnight with the primary antibody diluted with $5 \%$ skim milk powder (dilution, $1: 1,000$ ) at $4^{\circ} \mathrm{C}$; then washed with PBST 3 times the next day, 5 min each time; after incubated with HRP-labeled goat anti-mouse secondary antibody diluted with 5\% skim milk powder (dilution, 1:2,000) for $2 \mathrm{~h}$ at room temperature, wash 3 times with PBST, $5 \mathrm{~min}$ after each time, the chemiluminescence substract was applied to the PVDF membrane to develop color, and actin was used as an internal reference. Image $\mathbf{J}$ software (National Institute of Mental Health) was used to perform quantitative analysis.

Statistical analysis. All data were analyzed by SPSS 17.0 statistical software (SPSS, Inc., Chicago, IL, USA). The measurement data were expressed by mean \pm standard deviation (mean $\pm \mathrm{SD}$ ), and the measurement data were compared by Chi-square test. ANOVA was used for comparison of multiple groups with LSD test. $\mathrm{P}<0.05$ indicated that the difference was statistically significant.

\section{Results}

Comparison of behavioral indicators after treatment in each group of mice. Compared with the control group, the immobility time of the mice in the model group was significantly prolonged $(\mathrm{P}<0.05)$ in the tail suspension and forced swimming experiment, while the consumption of syrup water was significantly decreased at $24 \mathrm{~h}$, with a statistical difference $(\mathrm{P}<0.05)$. 
Table II. Comparison of DA, 5-HT and BDNF levels in each group of mice after treatment.

\begin{tabular}{llll}
\hline Groups & DA(ng/g) & $5-H T(n g / g)$ & BDNF(ng/g) \\
\hline Control & $654.39 \pm 30.19$ & $719.25 \pm 43.12$ & $21.43 \pm 6.17$ \\
Model & $198.25 \pm 21.44^{\mathrm{a}}$ & $300.18 \pm 21.44^{\mathrm{a}}$ & $8.43 \pm 3.18^{\mathrm{a}}$ \\
Low-dose & $298.33 \pm 23.56^{\mathrm{b}}$ & $381.24 \pm 25.44^{\mathrm{b}}$ & $17.11 \pm 4.21^{\mathrm{b}}$ \\
Medium-dose & $357.19 \pm 28.14^{\mathrm{b}, \mathrm{c}}$ & $430.29 \pm 28.10^{\mathrm{b}, \mathrm{c}}$ & $14.20 \pm 3.99^{\mathrm{b}, \mathrm{c}}$ \\
High-dose & $490.99 \pm 30.23^{\mathrm{b}-\mathrm{d}}$ & $551.33 \pm 32.55^{\mathrm{b}-\mathrm{d}}$ & $11.98 \pm 3.02^{\mathrm{b}-\mathrm{d}}$ \\
\hline
\end{tabular}

${ }^{\mathrm{a}} \mathrm{P}<0.05$ compared with the control group; ${ }^{\mathrm{b}} \mathrm{P}<0.05$ compared with the model group. ${ }^{\mathrm{C}}<0.05$ compared with the low-dose group; ${ }^{\mathrm{d}} \mathrm{P}<0.05$ compared with the medium-dose group.

Compared with the model group, the immobility time of the tail suspension experiment and the forced swimming experiment in the low-dose group was significantly shortened, while the 24-h sucrose consumption was significantly increased, and the difference was statistically significant $(\mathrm{P}<0.05)$. Compared with the low-dose group, the immobility time of the suspension experiment and forced swimming experiment in the middle dose group was significantly reduced, while the $24 \mathrm{~h}$ syrup consumption was significantly increased, the difference was statistically significant $(\mathrm{P}<0.05)$. Compared with the middle dose group, the immobility time of the tail suspension experiment and forced swimming experiment in the high dose group was significantly reduced, while the 24-h sucrose consumption was significantly increased, and the difference was statistically significant $(\mathrm{P}<0.05)($ Table I).

Comparison of DA, 5-HT and BDNF levels in each group of mice after treatment. We further analyzed the effects of resveratrol on neurotransmitter levels in the mice brain tissue. The ELISA results showed that the levels of DA, 5-HT and BDNF in the brain tissue of the model group were significantly downregulated compared with the control group, and the difference was statistically significant $(\mathrm{P}<0.05)$. After treatment with low-dose resveratrol, the levels of DA, 5-HT and BDNF in brain tissue were significantly higher than those in the model group, and the difference was statistically significant $(\mathrm{P}<0.05)$. Compared with the low-dose group, the levels of DA, 5-HT and BDNF in the brain tissue of mice treated with medium dose of resveratrol were significantly increased $(\mathrm{P}<0.05)$. Compared with the middle dose group, the levels of DA, 5-HT and BDNF in the brain of the high dose group were significantly increased, and the difference was statistically significant $(\mathrm{P}<0.05)$ (Table II).

Comparison of NPY levels in brain tissue of each group of mice. The expression levels of total NPY in the brain tissue of each group of mice were analyzed by western blot analysis. The results are shown in Fig. 1. Compared with the control group, the NPY levels of the model group were significantly downregulated, and the difference was statistically significant $(\mathrm{P}<0.05)$. After treatment with resveratrol, the level of NPY in the brain tissue of mice increased gradually, and in a dose-dependent manner. The comparison between the levels

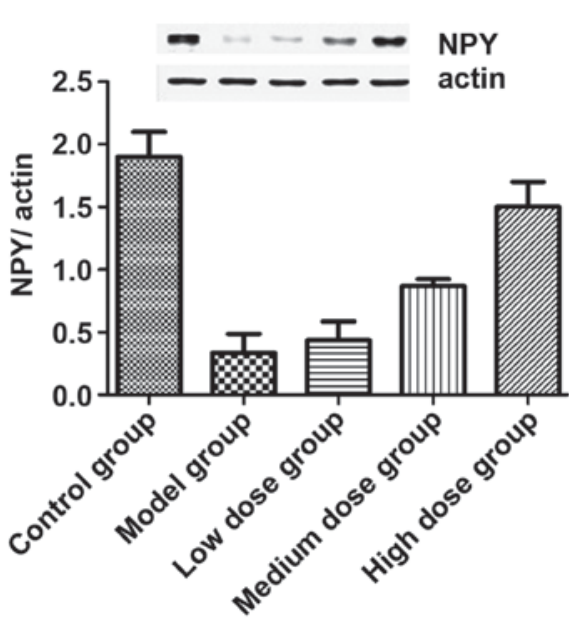

Figure 1. Comparison of NPY levels in brain tissue of each group of mice.

of NPY in the brain tissue of the low-dose, medium-dose and high-dose groups were significantly different $(\mathrm{P}<0.05)$.

\section{Discussion}

With the gradual increase of social and work pressure, depression, a chronic neurological disease, seriously threatens human cognition. Its incidence is increasing year by year. Epidemiological analysis shows that the incidence of different depression in young individuals is more significant and has become a social problem (11). Therefore, screening for highly effective and low-toxic drugs is a prerequisite for the treatment of depression. Our previous in vitro studies showed that after treatment of neurons with resveratrol, the level of dopamine was significantly increased. The study showed that the levels of DA, 5-HT and BDNF in patients with depression were significantly reduced, which is one of the main causes of depression. Therefore, we speculated whether resveratrol has the effect of relieving depression.

DA and 5-HT are monoamine neurotransmitters in the brain. In the pathogenesis of depression, the DA and 5-HT hypotheses are recognized. Studies also showed that the level of DA and 5-HT decreased in the hypothalamic tissue of patients with depression, indicating that depression is closely related to the low function of DA and 5-HT $(12,13)$. The levels of DA and 5-HT in the brain tissue of the mouse model of depression established in this study were significantly lower than those in normal mice, indicating that DA and 5-HT play an important role in the pathogenesis of depression. After treatment with resveratrol, the levels of DA and 5-HT increased gradually and in a dose-dependent manner. This result suggests that resveratrol may have the effect of treating depression. BDNF is a member of the neurotrophic factor family, which has the effect of promoting proliferation and differentiation of neurons, promoting neuronal survival and development, altering neuronal morphology in the brain, increasing synaptic terminal density, and promoting dendritic and axon growth (14). NPY is a known neuroendocrine polypeptide that plays an important regulatory role in neuronal excitability (15). The current study indicated that BDNF levels and brain NPY were significantly decreased in patients with depression. This result is consistent with the results obtained in 
the mouse model of this study. After treatment with resveratrol, the level of BDNY increased with the increase of resveratrol concentration, indicating that resveratrol may increase the activity of neurons by increasing brain-derived neurotrophic factor, thus achieving anti-depressant effect.

Behavioral analysis of mice showed that the behavior of depression in mice after resveratrol treatment was significantly relieved, indicating that resveratrol has a role in alleviating depression. Early studies on resveratrol have shown that they have important biological functions such as anti-inflammatory, antitumor and anti-aging. In addition, this study revealed a new anti-depressant biological function of resveratrol, which expanded the clinical use of resveratrol.

In summary, resveratrol can significantly increase the levels of DA, 5-HT, BDNF and NPY in the brain to achieve the effect of treating depression.

\section{Acknowledgements}

Not applicable.

\section{Funding}

No funding was received.

\section{Availability of data and materials}

The datasets used and/or analyzed during the present study are available from the corresponding author on reasonable request.

\section{Authors' contributions}

ZG conceived the study and drafted the manuscript. ZG and LC were responsible for construction and treatment of the depression models. ZG and YH helped with the sucrose consumption test. All authors have read and approved the final manuscript.

\section{Ethics approval and consent to participate}

The study was approved by the Ethics Committee of The Second Affiliated Hospital of Xinxiang Medical University (Xinxiang, China).

\section{Patient consent for publication}

Not applicable.

\section{Competing interests}

The authors declare that they have no competing interests.

\section{References}

1. Bennett S and Thomas AJ: Depression and dementia: Cause, consequence or coincidence? Maturitas 79: 184-190, 2014.

2. Korczyn AD and Halperin I: Depression and dementia. J Neurol Sci 283: 139-142, 2009.

3. Liu MY, Zhang LJ,Zhou YX and Wei WL: 5-Hydroxytryptamine changes under different pretreatments on rat models of myocardial infarction and/or depression. Chin Med J (Engl) 130: 2219-2225, 2017.

4. Teo CH, Soga T and Parhar IS: Brain beta-catenin signalling during stress and depression. Neurosignals 26: 31-42, 2018.

5. Martocchia A, Curto M, Scaccianoce S, Comite F, Xenos D, Nasca C, Falaschi GM, Ferracuti S, Girardi P, Nicoletti F, et al: Effects of escitalopram on serum BDNF levels in elderly patients with depression: A preliminary report. Aging Clin Exp Res 26: 461-464, 2014

6. Wu X, Xu Y, Zhu B, Liu Q, Yao Q and Zhao G: Resveratrol induces apoptosis in SGC-7901 gastric cancer cells. Oncol Lett 16: 2949-2956, 2018.

7. Li J, Xie C, Zhuang J, Li H, Yao Y, Shao C and Wang H: Resveratrol attenuates inflammation in the rat heart subjected to ischemia-reperfusion: Role of the TLR4/NF- $\kappa B$ signaling pathway. Mol Med Rep 11: 1120-1126, 2015.

8. Tan L, Wang W, He G, Kuick RD, Gossner G, Kueck AS, Wahl H, Opipari AW and Liu JR: Resveratrol inhibits ovarian tumor growth in an in vivo mouse model. Cancer 122: 722-729, 2016.

9. Tresguerres IF, Tamimi F, Eimar H, Barralet J, Torres J, Blanco L and Tresguerres JA: Resveratrol as anti-aging therapy for age-related bone loss. Rejuvenation Res 17: 439-445, 2014.

10. Subramanian M, Goswami M, Chakraborty S and Jawali N: Resveratrol induced inhibition of Escherichia coli proceeds via membrane oxidation and independent of diffusible reactive oxygen species generation. Redox Biol 2: 865-872, 2014.

11. Miniati M, Callari A, Calugi S, Rucci P, Savino M, Mauri M and Dell'Osso L: Interpersonal psychotherapy for postpartum depression: A systematic review. Arch Women Ment Health 17: 257-268, 2014.

12. Segura-Aguilar J, Paris I, Muñoz P, Ferrari E, Zecca L and Zucca FA: Protective and toxic roles of dopamine in Parkinson's disease. J Neurochem 129: 898-915, 2014.

13. Li X, Fan Y, Xiao S, Peng S, Dong X, Zheng X, Liu CC, Li H and Xiao Z: Decreased platelet 5-hydroxytryptamin (5-HT) levels: A response to antidepressants. J Affect Disord 187: 84-90, 2015.

14. Wilkinson ST, Kiselycznyk C, Banasr M, Webler RD, Haile C and Mathew SJ: Serum and plasma brain-derived neurotrophic factor and response in a randomized controlled trial of riluzole for treatment resistant depression. J Affect Disord 241: 514-518, 2018.

15. Sun Z, Liu S, Kharlamov EA, Miller ER and Kelly KM: Hippocampal neuropeptide Y protein expression following controlled cortical impact and posttraumatic epilepsy. Epilepsy Behav 87: 188-194, 2018. International (CC BY-NC-ND 4.0) License. 